\title{
Antioxidant enzyme activities of plants under conditions of combined temperature and viral stress
}

\author{
Amanbayeva U.I.*, Bekturova A.Zh., Tleukulova Zh.B., Kurmanbayeva A.B., \\ Gadilgereyeva B.Zh., Zhangazin S.B., Omarov R.T., Masalimov Zh.K. \\ L.N. Gumilyov Eurasian National University, Nur-Sultan, Kazakhstan \\ * e-mail: amanbayeva.ulbike@gmail.com
}

Temperature is one of the important environmental factor influencing plant development in natural and diseased conditions. Reactive oxygen species (ROS) are produced as a result of environmental stresses such as temperature and viral infection. There are limited information about antioxidant enzyme activities upon combined abiotic and biotic stress factors. The aim of this work was to investigate the influence of combined temperature and viral infection on aldehyde oxidase (AO) and catalase (CAT) activities in Nicotiana benthamiana plants.

A 24-h experiment of cold and heat stress was performed with plants, 10 and $40{ }^{\circ} \mathrm{C}$ respectively. After that one part of $N$. benthamiana plants were infected with Tomato bushy stunt virus (TBSV), other part were treated with virus-free buffer. Mock-inoculated plants in room temperature treated with virus-free buffer were usedas controls.

According to our results, under abiotic stress (room, cold and heat stresses) CAT activity was significantly higher in non-infected plants compared to infected plants. Imposition of heat stress had no significant impact on CAT activity in non-infected plants, whereas cold stress showed a decrement of this enzymatic activity. In response to individual temperature stress, AO activity did not change with respect to control values in leaves. Interestingly, under viral infection, CAT activity decreased, but AO activity increased in $N$. benthamiana plants compared to control levels. The combination of viral infection and temperature stresses negatively impacted on plants but the activation of the antioxidant machinery was associated to the ability to tolerate this stress combination.

As a result, in Nicotiana benthamiana plants the increment of AO activity along with the decline in CAT activity compared to control values could be partially responsible of its increased oxidative damage and sensitivity to the combination viral infection and high and low temperatures. Thus, Nicotiana benthamiana plants has the ability of efficiently coordinate $\mathrm{AO}$ and CAT activities involved in ROS detoxification. 